\title{
Screening of Escherichia coli 0157:H7 contamination and nitrate content on Butterhead lettuce from market outlets
}

\begin{abstract}
Escherichia coli O157:H7 is classified as biological hazard that can produce a bloody diarrhea due to its toxins. While nitrate in vegetables is classified as a chemical hazard if ingested greater than limit and risk getting gastrointestinal cancer and methemoglobinaemia. A study was conducted to obtain the information on E. coli O157:H7 contamination and nitrate content in Butterhead lettuce from local suppliers. The number of colonies E. coli O157:H7 from the lettuce was enumerated using standard plate count and the nitrate content was measured using nitrate meter. From the experiment, it was found that E. coli O157:H7 contamination of Butterhead lettuce in Malaysia is still within the safe limit for the consumers while the nitrate content was distributed differently between leaf structures; midrib, outer adult leaf blade and young leaves. Lettuce midrib and outer adult leaf blade had a higher concentration of nitrate compared to the young leaves.
\end{abstract}

Keyword: Escherichia coli O157:H7; Nitrate; Butterhead lettuce 